\title{
Efficient H.264/AVC video encoder where Pattern is Used as Extra Mode for Wide Range of Video Coding
}

\author{
Manoranjan Paul and Manzur Murshed \\ Gippsland School of IT, Monash University, Churchill Vic 3842, Australia \\ E-mail: \{Manoranjan.Paul,Manzur.Murshed\}@infotech.monash.edu.au
}

\begin{abstract}
Pattern-based video coding representing moving regions in macroblock has very good potential for improved coding efficiency over existing standard H.264/AVC in the very low bit-rate range. However, the coding efficiency diminishes compared to H.264 with the bit rates because variable block sizes of H.264 for motion estimation and compensation are successfully selected for any bit rates using weighting between bit rate and image quality. The existing pattern-based video coding scheme could not handle this flexibility with bit rates due to its fixed size and lack of verification with other modes of H.264. In this paper we propose two large and one small pre-defined pattern sets and treat them as extra modes of H.264. We also investigate the weighting factor between bit rate and image quality compared to the existing one. The experimental results confirm the superiority of our approach compared to H.264 and other existing pattern-based video coding for not only the very low bit rate range but also wide range of bit rates.
\end{abstract}

Keywords: Video coding, pattern matching, moving regions.

\section{Introduction}

Recently H.264/AVC standard [6] introduced block based motion estimation (ME) and motion compensation (MC) coding system using variable-block size (from $16 \times 16$ to $4 \times 4$ ) to approximate the shape of the moving objects within the Macroblock (MB). It requires separate motion vector for each partition and the choice of partition size. The number of partition types has a significant impact on coding efficiency. Choosing larger partition sizes $(16 \times 16,16 \times 8,8 \times 16)$ requires relatively small number of bits for motion vectors and the type of partition at the expense of containing significant amount of bits in the motion compensated residual error in areas of high detail. On the contrary, choosing smaller partition sizes $(8 \times 4,4 \times 8,4 \times 4)$ may give a lower bits residual at the expense of larger number of bits for motion vectors and the type of partition. However, there would be two issues which cannot be successfully handled with this partitioning concept. First thing is that we observed that in low to mid range of bit rates most of the MBs are motion estimated and compensated using larger partitions so that variable block size concept is ineffective in this area. Second thig is

This research was supported under the Australian Research Council's Discovery scheme (project number DP0666456). 
that no block based standard video coding can exploit the intra-frame temporal redundancy which is static in success frames.

To address this problem, Fukuhara et $a l$. [1] first proposed pattern based coding using four MB-partitioning patterns of 128-pixels each. ME and MC was carried out on all eight possible 128-pixel partitions of an $\mathrm{MB}$ and the pattern with the lowest prediction error was selected. By treating identically each $\mathrm{MB}$, irrespective of its motion content, also resulted in a higher bit-rate being incurred for those MBs which contained only static background or had moving object(s), but with little static background. In such cases, the motion vectors for both partitions were almost the same and so only one could be represented.
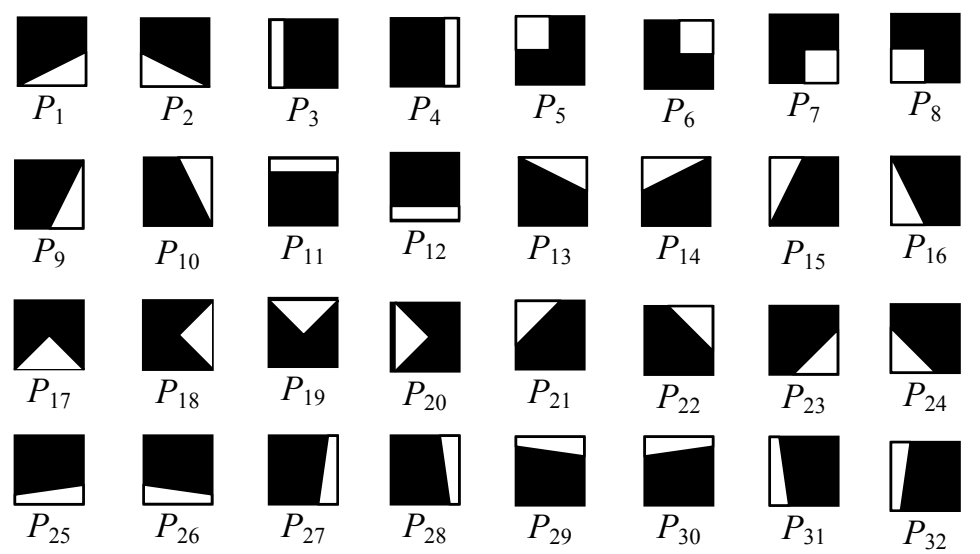

Figure 1: The pattern codebook of 32 regular shaped, 64-pixel patterns, defined in $16 \times 16$ blocks, where the white region represents 1 (motion) and black region represents 0 (no motion).

The MPEG-4 [4] video standard first introduced the concept of content-based coding, by dividing video frames into separate segments comprising a background and one or more moving objects. But for real-time application no content-based coding algorithm is a competitor of block-based coding due to the lack of automatic object segmentation algorithm. To address the limitations of [1], Wong et al. [11] exploited the idea of partitioning the MBs via a simplified segmentation process that again avoided handling the exact shape of moving objects, so that popular MB-based motion estimation techniques could be applied. Wong et al. [11] classified each MB into three distinct categories: 1) Static MB (SMB): MBs that contain little or no motion; 2) Active MB (AMB): MBs which contain moving object(s) with little static background; and 3) Active-Region $M B$ (RMB): MBs that contain both static background and part(s) of moving object(s). SMBs and AMBs are treated in exactly the same way as in H.26X. For RMB coding, Wong assumed that the moving parts of an object may be represented by one of the eight predefined patterns $P_{1}-P_{8}$ in Figure 1. An MB is classified as RMB if by using some similarity measure, the part of a moving object of an MB is well covered by a particular pattern. The RMB can then be coded using the 64 pixels of that pattern with the remaining 192 pixels being skipped as static background. Successful pattern matching can theoretically therefore have a maximum compression ratio of $4: 1$ for any MB. The actual achievable compression 
ratio will be lower due to the computing overheads for handling an additional MB type, the pattern identification numbering and pattern matching errors.

Figure 1 shows the complete 32-pattern codebook (PC), which forms the basis of the real time pattern selection (RTPS) algorithm [8]. The RTPS algorithm successfully introduced relevant measure together with pattern similarity measure to speed up the whole process with arbitrary size of pattern set without degrading the image quality.

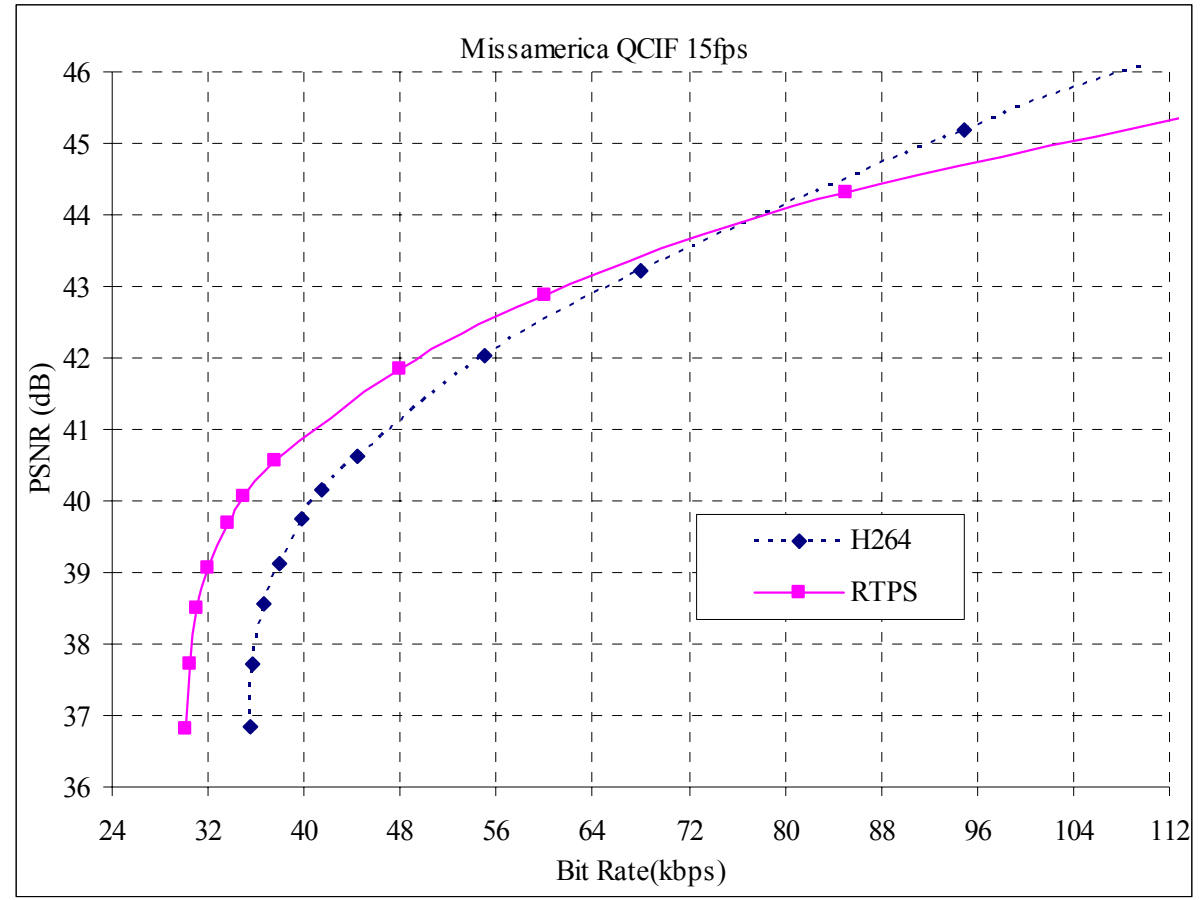

Figure 2: Rate-Distortion curves of the H.264 and RTPS using the QCIF Miss America video sequence.

The Figure 2 shows the comparison between the rate-distortion curves generated by the H.264 and RTPS (updated with H.264 features) algorithms using the Miss America video sequence. It is interesting to observe that for very low bit rate $(\leq 64 \mathrm{kbps})$ range the performance of the RTPS algorithm is better compared to the H.264 by around $1.0 \mathrm{~dB}$. But the performance of the RTPS is diminishing with bit rates against the H.264 and once the performance is worse.

To analysis the performance of RTPS algorithm, we easily observe that the moving region covered by the best-matched pattern template provides relatively less bits with better image quality; on the contrary, uncovered moving region provides poor image quality. Since at very low bit rate, a large scale distortion is occurred, thus image distortion due to the uncovered moving region is negligible compared to the high distortion in overall image. As a result, pattern-based video coding algorithm outperforms the H.264 standard for very low bit rate range. When the target bit rate is high, the distortion in uncovered moving region is relatively high compared to overall 
image distortion. As a result the rate-distortion performance of pattern-based coding diminishes with bit rates compared to the H.264.

To address this problem we proposed two ways, i) we consider pattern-based coding is as a mode, i.e., selected MB will be processed using pattern, and other variable block size modes, then we will pick that mode which provides us best ratedistortion performance. The reason behind of this approach is that when a pattern can not provide the best rate-distortion performance, the encoder automatically selects the best mode among the variable blocks so that it ensures the performance better or at least the same as the H.264 does, ii) we consider two large pattern sets with larger variable block size modes and one small size pattern set with small modes to ensure the approximation of the variable size moving regions more accurately.

This paper is organized as follows. The video coding strategy using the Conventional Pattern-based algorithm is described in Section 2, while some our proposed approach is described in Section 3. Analyses of simulation results are given in Section 4. Some future works and conclusions are provided in Section 5.

\section{Conventional Pattern based Video Coding}

Let $C_{k}(x, y)$ and $R_{k}(x, y)$ denote the $k^{\text {th }} \mathrm{MB}$ of the current and reference frames, each of size $W$ pixels $\times H$ lines, respectively of a video sequence, where $0 \leq x, y \leq 15$ and $0 \leq k<W / 16 \times H / 16$. The moving region $M_{k}(x, y)$ of the $k^{\text {th }} \mathrm{MB}$ in the current frame is obtained as follows:

$$
M_{k}(x, y)=T\left(\left|C_{k}(x, y) \bullet B-R_{k}(x, y) \bullet B\right|\right)
$$

where $B$ is a $3 \times 3$ unit matrix for the morphological closing operation $\bullet$ [2][7], which is applied to reduce noise, and the thresholding function $T(v)=1$ if $v>2$ and 0 otherwise.

Let $|Q|_{\ell}$ be the total number of $\ell$ 's in the matrix $Q$. Similarity of a pattern $P_{n} \in$ $\mathrm{PC}$ with the moving region in the $k^{\text {th }} \mathrm{MB}$ can be defined efficiently [9] as

$$
S_{k, n}=\left|M_{k}\right|_{1}-\left|M_{k} \wedge P_{n}\right|_{1} \text {. }
$$

Clearly, higher the similarity lower will be the value of $S_{k, n}$. Obviously all MBs are not examined using pattern. The eligible MBs are called candidate RMB (CRMB) and defined as $8 \leq\left|M_{k}\right|_{1}<T_{S}+$ PatternSize, where $T_{S}$ and PatternSize are the predefined similarity threshold and one of $\{16,64,128\}$ respectively. The candidate $\mathrm{CRMB}$ is classified as an RMB and its moving region is represented by a pattern $P_{i}$ such that

$$
\left.P_{i}=\arg \min _{\forall P_{n} \in \mathrm{PC}}\left(S_{k, n}\right) \mid S_{k, n}<T_{S}\right)
$$

otherwise, the CRMB is classified as an AMB. For a given PC, an image sequence is coded using the general pattern based coding $(\mathrm{PBC})$. To avoid more than four $4 \times 4$ block of DCT calculations for 64 residual error values per RMB, these values are 
rearranged into an $8 \times 8$ block. It avoids unnecessary DCT block transmission. A similar inverse procedure is performed during the decoding.

By this conventional pattern-based coding approach, if a MB is classified as CRMB and the similarity threshold permits it to classify as a RMB, then this MB is finally classified as RMB irrespective of its rate-distortion performance compared to the other available modes. This strategy performs very well when the operational target bit rate very low. As mentioned in earlier, the moving region covered by the best-matched pattern template is motion estimated and compensated and the rest of the region is treated as static region which is simply copied from the reference MB in the decoder end. Since the uncovered region is three times of the pattern template covered region, successful pattern matching can theoretically therefore have a maximum compression ratio of 4:1 for RMB. It is obvious that sometimes a tiny portion of object may remains in so called static region which is considered in pattern-based video coding. This tiny portion of object has little influence in very low bit rate range as significant amount of image distortion is occurred due to the course quantization in overall image. However, this tiny portion of object may have significant impact when the operational bit rate is relatively high where finer quantization is used to improve the image quality. In this case rate-distortion performance of RMB may not be better than that of other modes. As a result the overall coding performance would degrade.

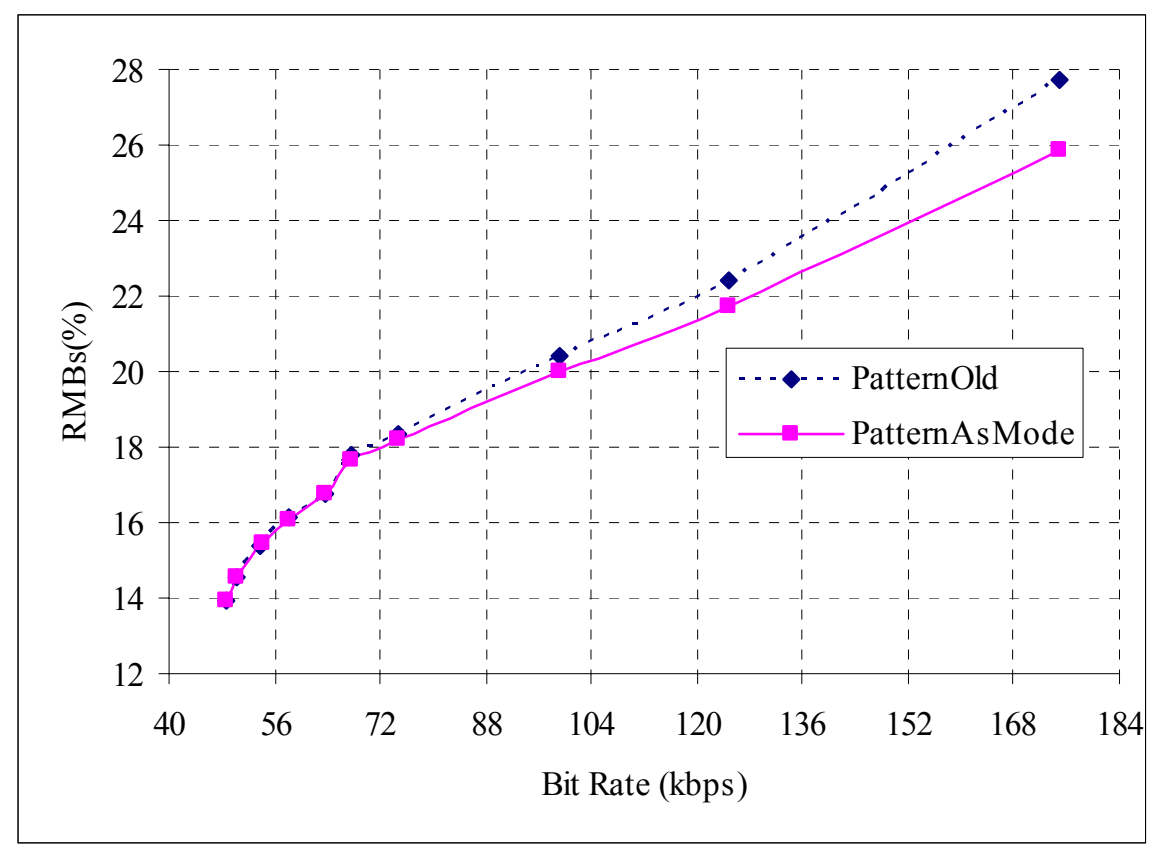

Figure 3: Percentage of RMBs classified using conventional PBC (PatternOld) and PBC as one of H.264 modes (PatternAsMode) using a number of QCIF video sequences against bit rates. 
The experimental results reveal that using conventional approach of $\mathrm{PBC}$, the percentage of SMB and MBs classified by larger modes (e.g., $16 \times 16,16 \times 8,8 \times 16$ ) decreases and RMB increases with bit rates. After a certain bit rate, when small modes are dominant modes, the increase of RMB should decrease for better ratedistortion performance. The conventional PBC could not do it because its ratedistortion performance is not compared to the other modes for final mode selection decision. Simply if we compare the rate-distortion performance of PBC with other rate-distortion performance generated by other modes and then finally we select minimum one, the overall performance will be better. The percentage of RMB using both conventional PBC (PatternOld) and PBC as a mode (PatternAsMode) is shown in Figure 3. The figure shows that within 60 to $80 \mathrm{kbps}$ bit rates, the percentage of RMB using PatternAsMode is decreasing compared to the PatternOld. Later we will show that within that bit rate range, the performance of PatternAsMode is better than PatternOld.

\section{Our Approach}

The method of Lagrange multipliers (LM) is used to trade off between the quality of the compressed video and the bit rate generated for different modes. In this method, the LM $(\lambda)$ is first calculated with an empirical formula using the selected Quantization Parameter (QP) for every MB [4]:

$$
\lambda=0.85 \times 2^{\frac{(Q P-12)}{3}} .
$$

During the encoding process, all possible modes (actually $16 \times 16,16 \times 8,8 \times 16$, $8 \times 8$ are first examined if $8 \times 8$ is selected then examined $8 \times 4,4 \times 8,4 \times 4$, otherwise not) of every $\mathrm{MB}$ are examined and the resulting rates, $R\left(m_{i}\right)$ and the distortions, $D\left(m_{i}\right)$ are determined, where $m_{i}$ is the $i$-th $(i=1 \cdots 7)$ mode. The Lagrangian cost function is defined as:

$$
J^{L M}\left(m_{i}\right)=D\left(m_{i}\right)+\lambda \times R\left(m_{i}\right) .
$$

where $R\left(m_{i}\right)$ is the sum of the bits for mode $m_{i}$, including the mode information, the motion vectors and the transformation coefficients, while $D\left(m_{i}\right)$ is measured as the Sum of Square Difference (SSD) between original MB and corresponding reconstructed $\mathrm{MB}$ for mode $m_{i}$.

The mode $m_{n}$ is selected as follows:

$$
m_{n}=\arg \min _{\forall m_{i}}\left(J^{L M}\left(m_{i}\right)\right) R\left(m_{i}\right) \leq R^{T}
$$

where $R^{T}$ is the target bit rate.

We also investigate the LM value by introducing the pattern mode. From our experimental results (see Figure 4) we observed that the relationships between QP and LM are almost same for H.264 and H.264 with pattern as an extra mode using Miss 
America sequence. Same relationship is also observed for different video sequences. Thus, we conclude that no modification is required in LM value, as the relationship between the LM and QP remains same after successfully introducing the extra pattern mode.

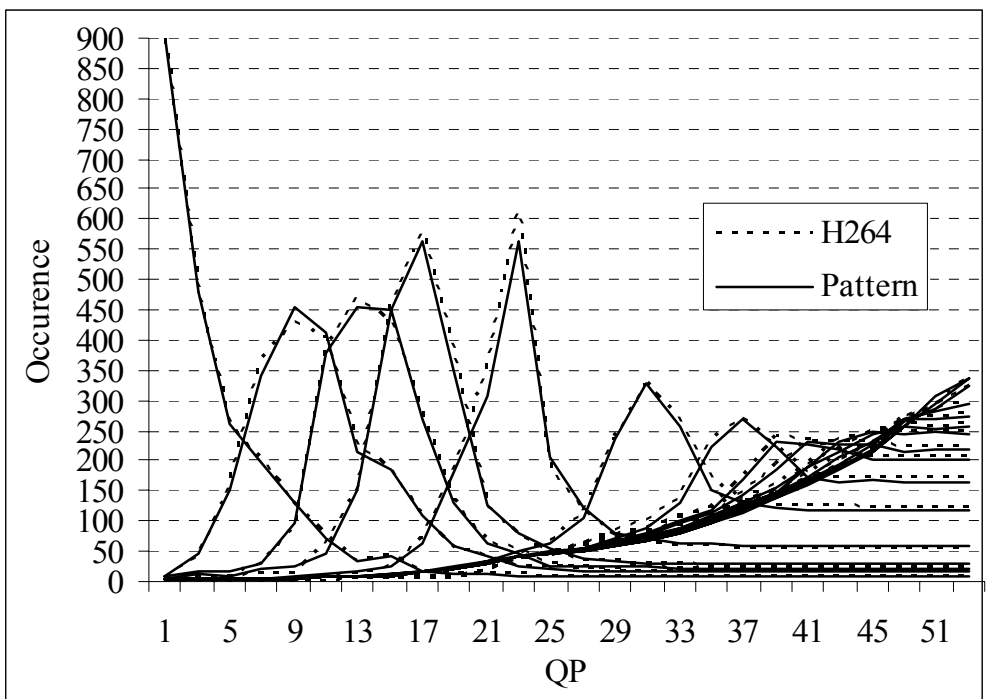

Figure 4: Occurrence vs. macroblock QP for various Lagrange parameters $(0.050 .25$ 0.5142510025040073010001500250040006000 10000). The occurrence of macroblock QP values gathered while coding first 100 frames of the Miss America video sequence using H.264 itself (H264) and H.264 with pattern as an extra mode (Pattern).

In our approach we proposed three sets of patterns of 128-pixel, 64-pixel, and 16-pixel. The 64-pixel 32 patterns are same as Figure 1, and other two sets are given in Figure 5. We do not claim that this choice of shapes and number of patterns in PC set is the best but simply observe that, with this arbitrary choice of PCs, our algorithm provides better performance compared to the existing algorithm. It is left for future work to determine the best PC sets.

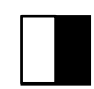

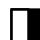
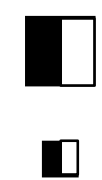
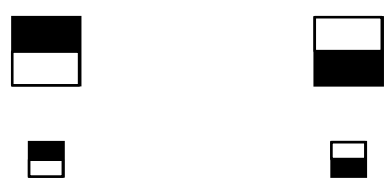

Figure 5: The pattern codebook of four regular shaped, 128-pixel patterns, defined in $16 \times 16$ blocks in top row and 16-pixel patterns defined in $8 \times 8$ blocks in bottom row, where the white region represents 1 (motion) and black region represents 0 (no motion).

In our approach we determine extra two and one Lagrangian cost function using bit rates and distortions generated by PBC algorithm using 64- and 128-pixle pattern 
sets for large modes and 16-pixel pattern set for small modes respectively. In encoded process each CRMB is motion estimated and compensated using 64- and 128-pixel pattern sets together with the large modes (from $16 \times 16$ to $8 \times 8$ ) and then based on the minimum Lagrangian cost function, we select one mode. If $8 \times 8$ mode is selected then each CRMB is again motion estimated and compensated using 16-pixel pattern set together with small modes (from $8 \times 4$ to $4 \times 4$ ). Based on the minimum Lagrangian cost function we select one mode.

\section{Simulation Results}

We have implemented our proposed algorithm based on the Baseline profile of H.264/AVC with full search motion estimation of maximum \pm 7.5 pixel search width [13] for a number of standard [10] video sequences with QCIF format namely, Foreman, Miss America, Claire, Car phone, News, Salesman, Suzie, and Grandma. But for brevity we show our rate-distortion performance using the first 100 frames of four standard video sequences. In this experiment, the GOP size is fifteen and we use only $I$ and $P$ frames. From now on, conventional pattern-based video coding, patterbased video coding as mode, and pattern-based video coding with three variable size PC sets is termed as PatternOld, PatternAsMode, and PatternVS.

The final rate-distortion performance is demonstrated in Figure 6 for four QCIF standard video sequences with 15 frames per second (fps). The experimental results reveal that at a wide range of bit rates the PBC using three different pattern set and pattern as a mode outperforms both the conventional PBC and the H.264 standard. For more specific, the PBC with three different PCs performs as high as $1.5 \mathrm{~dB}$ (for Salesman) at 100 kilo bit rates (kbps).

\section{Future Works and Conclusions}

Video coding using pattern templates representing moving regions in macroblock has already established its potentiality for improving coding efficiency over existing standard H.264/AVC in the very low bit-rate range. However, the coding efficiency diminishes compared to H.264 with the bit rates because variable block sizes of H.264 for motion estimation and compensation are successfully selected for any bit rates using weighting between bit rate and image quality. The existing pattern-based video coding scheme cannot handle this flexibility with bit rates due to its fixed size and lack of competition with other modes of H.264. To improve the performance in wider range of bit rates we treat pattern-based video coding as an extra mode and we also incorporate two large and one small pre-defined pattern sets in existing scheme. The experimental results confirmed that this new scheme improves as high as $1.5 \mathrm{~dB}$ image quality compared to the H.264 standard at the same bit rates. We do not claim that this choice of pattern set is the best but there would be other combination of pattern set provides better results. We observed that, with this arbitrary choice of pattern set, 
our algorithm provides better performance compared to the existing algorithm. It is left for future work to determine the best pattern set.
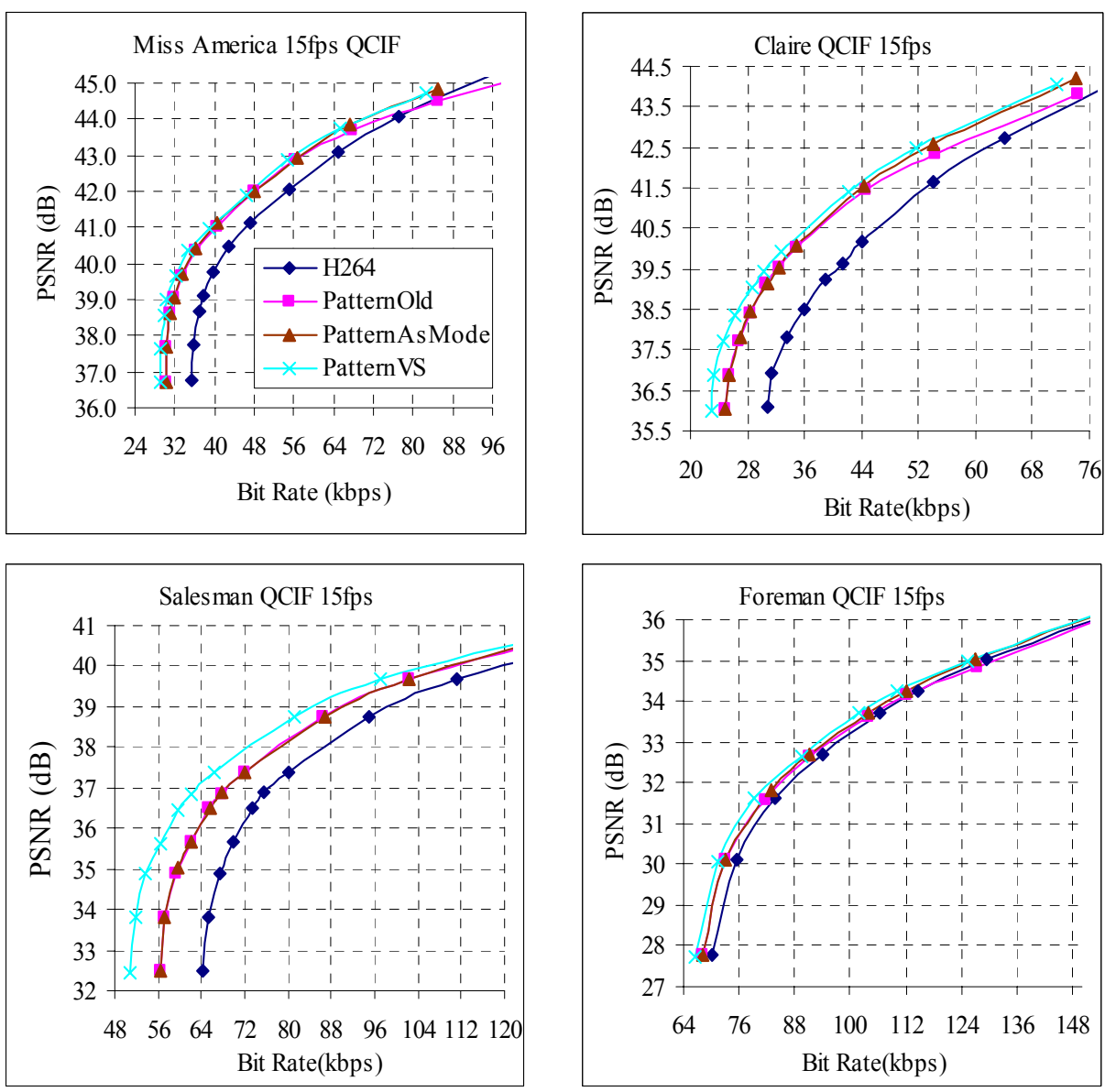

Figure 6: Coding performance comparisons for four standard test video sequences by the H.264, conventional PBC (PatternOld), PBC as mode of H.264 (PatternAsMode), and $\mathrm{PBC}$ with three different pattern sets (PatternVS).

\section{References}

[1] Fukuhara, T., K. Asai, and T. Murakami, "Very low bit-rate video coding with block partitioning and adaptive selection of two time-differential frame memories," IEEE Trans. Circuits Syst. Video Technol., 7, 212-220, 1997. 
[2] Gonzalez, R.C. and R. E. Woods, Digital Image Processing, Addison-Wesley, 1992.

[3] ISO/IEC 13818, MPEG-2 International Standard, 1995.

[4] ISO/IEC N4030, MPEG-4 International Standard, 2001.

[5] ITU-T Recommendation H.263, "Video coding for low bit-rate communication," Version 2, 1998.

[6] ITU-T Rec. H.264/ISO/IEC 14496-10 AVC. Joint Video Team (JVT) of ISO MPEG and ITU-T VCEG, JVT-G050, 2003.

[7] Maragos, P., "Tutorial on advances in morphological image processing and analysis," Opt. Eng., 26(7), 623-632, 1987.

[8] Paul, M., M. Murshed, and L. Dooley, "A real-time pattern selection algorithm for very low bit-rate video coding using relevance and similarity metrics," IEEE Trans. on Circuits and Systems on Video Technology, vol. 15, no. 6, pp. 753-761, June, 2005.

[9] Paul, M., Murshed, M. and Dooley, L., "A new efficient similarity metric generic computation strategy for pattern-based very low bit-rate video coding," Proc. of the IEEE Int. Con. of Acoustics, Speech, and Signal Proc. (ICASSP-04), vol. 3, pp. 165168, May, 2004.

[10] Shi, Y.Q. and H. Sun, Image and Video Compression for Multimedia Engineering Fundamentals, Algorithms, and Standards, CRC Press, 1999.

[11] Wong, K.-W., K.-M. Lam, and W.-C. Siu, “An Efficient Low Bit-Rate VideoCoding Algorithm Focusing on Moving Regions," IEEE trans. circuits and systems for video technology, 11(10), 1128-1134, 2001.

[12]Weigrand, T., H. Schwarz, A. Joch, and F. Kossentini, "Rate-contrained coder control and comparison of video coding standards," IEEE Trans. on Circuits and Systems on Video Technology, 13 (7), 688-702, 2003.

[13]Richardson, I. E. G., "H.264 and MPEG-4 video compression," Wiley Press, 2003. 